\title{
A Study On Factors Affecting Innovation Performance of Chinese Technology Sourcing Overseas M\&A Du Rui ${ }^{1,}$, , Li Lingzhi ${ }^{2, b}$ \\ ${ }^{1}$ Nanjing University of Science and Technology, Nanjing 210094, China; \\ ${ }^{2}$ Nanjing University of Science and Technology, Nanjing 210000, China. \\ apentadim@163.com, 'llzthy@126.com
}

Keywords: Technology Sourcing Overseas M\&A, Innovation Performance, Integrate

\begin{abstract}
With the rapid development of China's economy, Chinese enterprises develop fast. More and more Chinese enterprises try to get advanced technology by overseas M\&A. Consequently, it is necessary to study the factors affecting innovation performance of Chinese technology sourcing transnational M\&A. Using the related data by 2015, the paper analyzes the technology sourcing transnational M\&A deals initiated by Chinese enterprises over the years 2003-2012, providing a comprehensive analysis on the factors that affect the innovation performance. The results show that the smaller cultural difference is, the smaller absolute and relative knowledge scale of target enterprise is, the larger the magnitude of the merger is, the larger the technical knowledge base of the Chinese enterprise is, the better innovation performance will be. If these factors go in the opposite directions, the innovation performance of transnational M\&A deteriorates. The paper thus get some meaningful conclusions and revelations.
\end{abstract}

\section{Introduction}

In recent years, Chinese enterprise's overseas M\&A have been expanding. During the decade from 2006 to 2015, Chinese enterprise's overseas M\&A increase from 20 to 382, while the amount increases from $\$ 5.9$ billion to $\$ 67.4$ billion. With the accelerating process of economic globalization, Chinese enterprise has undergone major changes in the way of foreign investment. More and more enterprises choose transnational mergers and acquisitions to enter the international market.

Chinese enterprises aim at enhancing their innovation capabilities through overseas technology acquisition. In 2005, Sichuan Changhong bought Sterope at a price of $\$ 1.9$ billion to acquire the patents in the area of PDP. And then achieve breakthroughs and improve capability of independent innovation. In 2010, Geely's acquisition of Volvo Car Corporation for \$1.8 billion also boosted its technological innovation capability by continuously integrating the brand technical resources. These cases demonstrate that it is an effective way for Chinese companies to cross technologic barriers through technology sourcing transnational mergers and acquisitions. It is of great significance to the development of Chinese enterprise.

However, transnational merger is a dynamic and complex process. It contains great risk both before and after the merger. Thus, in order to improve the innovation performance of acquisition, what factors should enterprises consider? What kind of impact will these factors produce? Based on the empirical analysis, we study the factors affecting innovation performance of Chinese technology sourcing overseas M\&A, which is meaningful both in theory and practice for Chinese enterprise to implement transnational merger.

\section{Literature Review}

Technology sourcing overseas acquisition obtain technology and knowledge from target overseas enterprises who have advanced technology. It helps enterprise to increase knowledge storage and enhance the technological innovation capability of enterprise.

Foreign scholars have long been research on this subject. Ahuja and Katila (2001) has studied mergers and acquisitions in chemical industry in the United States. They find that the absolute scale of knowledge will enhance innovation performance while the relative scale has a negative effect on 
innovation performance. Based on Ahuja's research, Cloodt M, Hagedoom J and Van Kranenburg H (2006) do empirical research on four high-tech industries----air defense manufacturing, computer and office equipment manufacturing, medicine industry and telecom industry. The research shows that the absolute scale of knowledge has positive effect on innovation performance, but will gradually change to negative effect as time goes on. Gelinsichuan (1998) discusses the role of mergers and acquisitions for companies to acquire external technology, considering mergers and acquisitions has an important role for the introduction of technology.

Domestic scholars also have a lot of related research. Fang Lin, Song Dahai (2007) analyze the success factors as well as the core technology of transnational mergers and acquisitions. The results show that experience and financial support is essential for the success of transnational mergers and acquisitions. But most companies do not have these conditions, resulting in success rate of transnational merger and acquisition is still in the lower level. Zhou Xueping (2007) has studied the effects of transnational merger and acquisition on enterprises in China by examining a specific case----Wanxiang Group, which proves that merger does have promoting effect on technological progress. Wang Tianyi, Tang Shizhang (2007) finds that more and more enterprises tend to obtain advanced technologies and upgrade their technology skills by mergers and acquisitions based on the current situation of manufacturing enterprise's overseas M\&A.

Most existing research have studied whether transnational M\&A would help to improve enterprise's technology innovation capability. Few research about comprehensive factors influencing the innovation performance of technology acquisition. Thus we want to do some research from this perspective, trying to explore the effect of different factors.

\section{Variables and Hypothesis}

Technology sourcing transnational merger will be influenced by all kinds of complex factors. Through widely collecting related information, combining Chinese enterprise's specific situation, we finally determine the variables used in the empirical analysis: (1) culture distance; (2) absolute knowledge scale of target enterprise; (3) relative knowledge scale of target enterprise; (4) scale of merger; (5) base of technology; (6) age of enterprise. Specific reasons for selection of the variables and hypothesis explain as follows:

\section{(1) Culture distance}

Enterprises may face conflicts between different cultures in overseas mergers and acquisitions. Culture integration is a big challenge to the innovation performance of overseas acquisition. It is an important issue to reduce barriers caused by cultural difference in the process of technology integration. Greater cultural distance between different countries will correspondingly lead to more difficulties in integration. Also the innovation performance of acquisition will be affected. We assume that:

H1: Other things being equal, the smaller cultural difference is, the better innovation performance will be.

\section{(2) Absolute knowledge scale of target enterprise}

Different from traditional mergers, the economy of scale of technology acquisition is mainly reflected in R\&D activities. Through the integration, enterprise can reduce duplication of R\&D activities to lower the cost of research and development. In addition, foreign scholar's study shows that a lot of innovations are produced by recombining existing knowledge and technology. The bigger the knowledge scale of target enterprise is, the more synergies will be produced. We assume that:

H2: Other things being equal, the bigger absolute knowledge scale of target enterprise is, the better innovation performance will be.

(3) Relative knowledge scale of target enterprise

The efficiency of integration depends on the base of technology and knowledge of both sides. The bigger relative knowledge scale is, the more difficult the integration will be. We assume that:

H3: Other things being equal, the smaller relative knowledge scale of target enterprise is, the better innovation performance will be. 


\section{(4) Scale of merger}

The scale of merger is the embodiment of the value of technology. It is also directly related to the cost and the expected performance. We assume that:

H4: Other things being equal, the bigger the scale of merger is, the better innovation performance will be.

\section{(5) Base of technology}

Based on its own technology, enterprise can integrate target enterprise. It will be better for enterprise to integrate if it has more resources of technology and knowledge. Thus facilitate innovation performance. We assume that:

H5: Other things being equal, the better base of technology enterprise owns, the better innovation performance will be.

\section{(6) Age of enterprise}

Enterprises need a certain level of experience and competence to face the complex situation in overseas acquisitions. As business grows, the accumulated experience will form part of the intangible assets. In technology sourcing overseas M\&A, experience is beneficial for enterprise to deal with many problems and improve the innovation performance. We assume that:

H6: Other things being equal, the longer enterprise runs, the better innovation performance will be.

\section{Model, Samples and Data collection}

\subsection{Model}

We construct the following linear regression model to examine the factors affecting innovation performance of Chinese enterprise's technology sourcing overseas M\&A:

$$
M I P_{i}=\beta_{0}+\beta_{1} C D_{i j}+\beta_{2} A K S_{i}+\beta_{3} R K S_{i}+\beta_{4} S C_{i}+\beta_{5} B A_{i}+\beta_{6} A G_{i}+\varepsilon_{i}
$$

In the model, Subscript $i$ represents enterprise $i$, dependent variable ${ }^{M I P_{i}}$ represents the innovation performance of enterprise $i$, which is measured by the number of patents within three years after the merger; $C D_{i j}$ represents the culture distance between target enterprise and enterprise $i$, that is the culture distance between China and country $j$ of target enterprise. Here we use Hofstede index to measure cultural distance. It is composed of power distance, uncertainty avoidance, individualism/collectivism, masculinity/femininity, short-term/long-term orientation and indulgence/restraint. The formula is as follows:

$$
C D_{i j}=\sqrt{\sum_{m=1}^{6}\left\{\left(I_{m j}-I_{m c}\right)^{2} / V_{m}\right\}}
$$

In the formula above, $I_{m j}$ represents the $m$ cultural dimension index of target country $j$, $I_{m c}$ represents the $m$ cultural dimension index of China, $V_{m}$ represents variance, the smaller $C D_{i j}$ is, the smaller culture distance is; $A K S_{i}$ represents the absolute knowledge scale of target enterprise, which is measured by patents of target enterprise within the 5 years before the merger; $R K S_{i}$ represents the relative knowledge scale of target enterprise, which is measured by the ratio of target enterprise and enterprise $i$,s patents within the 5 years before the merger; $S C_{i}$ represents the scale of merger, which is measured by transaction amount; ${ }^{B A_{i}}$ represents the base of technology of enterprise $i$, which is measured by the number of patents within 5 years before the merger; $A G_{i}$ represents the age of enterprise $i$, which is measured by the time since it sets. 


\subsection{Samples and Data collection}

We select samples according to the standard that whether the acquisition is aimed at gaining technology. According to the filter criteria and the availability of data, we sort out 39 technology sourcing overseas acquisitions of China's enterprises between 2003-2012. Since the measurement of innovation performance requires a time of 3 years, the samples do not include the acquisition of 2013-2015. We collect these merger cases from CSMAR, ZEPHYR, company's Website and so on. And the patent information from Search.cnipr.com and the State intellectual property office of the P.R.C.

Seeing from the countries and regions where target enterprises from, the samples involve 12 countries or regions, Germany and the United States account for the largest proportion, respectively, $33.33 \%$ and $30.77 \%$. From the view of target enterprise industry, mainly are machinery, electronic information, automobile, equipment and other industries.

\section{Analysis and Results}

We use SPSS 22 to do descriptive statistics and regression analysis, and the results are shown in the tables below. We can get the important factors affecting innovation performance of Chinese technology sourcing acquisition.

\subsection{Descriptive Statistics}

As shown in Table 1, the scale of overseas M\&A differs a lot. Also, some enterprise are young while others have run for many years. Besides, we can find differences in base of technology. From this table we can see there exist many different factors in transnational mergers and acquisitions.

Table 1 Three Scheme comparing

\begin{tabular}{cccccccc}
\hline Variable & MIP & CD & AKS & RKS & SC & BA & AG \\
\hline Min & 4.00 & 1.41 & 1.00 & 0.01 & 27.00 & 1.00 & 5.00 \\
Max & 7424.00 & 5.32 & 323.00 & 34.50 & 180000.00 & 3084.00 & 58.00 \\
Mean & 439.33 & 4.25 & 38.23 & 2.03 & 12356.76 & 282.21 & 21.18 \\
Standard Deviation & 1206.75 & 0.83 & 70.14 & 6.51 & 30316.99 & 581.36 & 15.40 \\
\hline
\end{tabular}

\subsection{Empirical Results}

It can be seen from table 2 that $C D$ has a weak positive correlation with innovation performance. It shows that the greater cultural difference is, the better innovation performance is, which is contrary to the original hypothesis. There are reasons, target enterprises in these acquisitions are mostly from American and European countries, while those countries and Chinese culture are quite different but their technology is generally more advanced. It is easier to gain advanced technology from enterprises in these countries. Thus the innovation performance is better though the culture distance is great.

From table 2 we can find that $A K S$ has a significant negative correlation with innovation performance. It shows that the bigger absolute knowledge scale of target enterprise is, the worse innovation performance is, which is contrary to the original hypothesis. There are reasons, though Chinese enterprises develop rapidly these years, the technical capacity of most enterprises is still weak. If the absolute knowledge scale of target enterprise is too big to integrate, it will have a negative impact on innovation performance. Indicator $R K S$ shows a significant negative correlation with innovation performance, which matches the original hypothesis. It shows smaller relative knowledge scale of target enterprise will lead to better innovation performance.

Table 2 Regression results analysis

\begin{tabular}{cccccccc}
\hline Variable & C & CD & AKS & RKS & SC & BA & AG \\
\hline P & 0.177 & $0.077^{*}$ & $0.001^{* *}$ & $0.002^{* *}$ & $0.000^{* * *}$ & $0.029^{* *}$ & 0.478 \\
Standardized Coefficient & -- & 0.065 & -0.139 & -0.145 & 0.877 & 0.182 & -0.026 \\
& & & & & & &
\end{tabular}

Note: *, **, *** mean significance on the level of $10 \%, 5 \%, 1 \% . \quad F=133.108(<0.0001), R^{2}=0.961, \quad$ Adj $R^{2}=0.954$ 
In addition, $S C$ has a significant positive correlation with innovation performance, which is consistent with the original hypothesis. Bigger scale of acquisition indicate that the technology is more valuable so it is easier for enterprise to improve innovation performance; $B A$ also has a significant positive correlation with innovation performance which shows that the technical knowledge ability of enterprise itself is the important foundation to obtain innovation performance; $A G$ does not show obvious correlation with innovation performance. Possibly because many young enterprises can integrate technology quickly while old enterprises work dilatorily.

From the analysis of regression coefficient, cultural differences impact the smallest, the scale of acquisition impact the most. Besides, technology knowledge base of acquirer, absolute and relative knowledge scale of targets also have great influence.

\section{Conclusions and Recommendations}

The research study factors affecting innovation performance of transnational mergers of Chinese enterprise based on 39 cases of the year 2003-2012. Through selecting explanatory variables which have strong correlation with technology acquisition, we test the relevance between these variables and innovation performance of transnational mergers. The research reveals how these factors influence innovation performance of transnational mergers of Chinese enterprises. The research has tried a new perspective and also can be complement to related research.

This study makes sense, it has shown that cultural differences have a weak impact on innovation performance of transnational merger of Chinese enterprises, which is inseparable from the specific situation in China. It indicates that Chinese enterprises still need to aim at enterprises in Europe and the United States as the target of transnational merger and acquisition.

The innovation performance is significantly influenced by absolute and relative knowledge scale of target enterprise, indicating that in order to improve the innovation performance of transnational mergers and acquisitions, Chinese enterprises need to choose the most suitable object. Overly large acquisition will increase the difficulty of integration and also greatly increase the risk.

In addition, the scale of merger has significantly positive correlation with innovation performance. This shows that large enterprises are more capable to guarantee innovation performance of transnational merger. For middle and small-sized enterprises in China, it remains a severe problem how to avoid risk and improve innovation performance in transnational merger; the research also shows the effect of technology base on innovation performance, which shows that Chinese enterprise still need to strengthen itself to upgrade its capacity of technology innovation.

Through this research, we recognize that Chinese enterprises need to choose the right goals, clear its direction and avoid risk in technology sourcing transnational mergers and acquisitions. Currently Chinese enterprises speed up the process of internationalization, but are still generally short of international management experience. The government should introduce relevant policies, laws and regulations to guide and encourage transnational merger of Chinese enterprises, and consequently promote the long-term development of Chinese enterprises' transnational mergers and acquisitions.

\section{References}

[1] Ying Huang. The Research on Practice and Strategy of Technology Sourcing Foreign Direct Investment in USA by Chinese Enterprises [J]. Science and Technology Management Research, 2014(9):86-90.

[2] Hu Hongyan. Research review on the transnational merger reason [J]. Economic Research Guide, 2014(14):226-229.

[3] He Zhiyong, Chen Wei. Research on the Comprehensive Evaluation of Risk Faced by Overseas M\&R Enterprise Based on the Target of Technology Acquisition [J]. Science \& Technology Progress and Policy, 2010(21):119-123. 
[4] Meng Fanchen, Miao Hui. The Correlation Analysis between Cross-border M\&A and Corporate Technological Progress of China [J]. Journal of Beijing Institute of Technology (Social Sciences Edition), 2010.12(2).

[5] Sun Chao, Shi Jingyang. Risk Strategy of Technology Sourcing Transnational Acquisition [J]. Communication of Finance and Accounting, 2013(8):113-114.

[6] Fang Lin, Song Dahai. Cross-border M\&A VS Core Technology [J]. Business Management, 2007(2):136-141.

[7] Wang Tianyi, Tang Shizhang. Reason of technology cross-border M\&A of Chinese manufacturing [J]. Journal of Anhui Agri. Sci., 2007, (35(10):3133-3134.

[8] Zhu Qin, Liu Yao. Empirical Analysis on Influencing Factors of Financial Performance of Cross-border Mergers by China's Listed Companies [J]. Journal of International Trade, 2013(8):151-169. 